\title{
Segmentation of PolSAR image by using an automatic initialized variational model and a dual optimization approach
}

\author{
Zongjie Cao*, Ying Tan and Jilan Feng
}

\begin{abstract}
This paper presents a variational model based segmentation approach for polarimetric synthetic aperture radar (PoISAR) images. The formulation for PoISAR image segmentation is based on a scaled Wishart distribution based continuous Potts model, which can partition the image domain into distinct regions with respect to the statistical property of PoISAR data. To make the segmentation efficient, a duality based optimization approach is utilized to minimize the energy functional. Moreover, an automatic initialization approach which takes the unsupervised $\mathrm{H}-\mathrm{a}$ classification result of the polarimetric data as input is used to initialize the segmentation process. This approach can estimate the appropriate number of clusters and the corresponding classification map for the PoISAR data, which are used as the input of the following variational segmentation approach. In such a way, the proposed approach is carried out in a fully unsupervised way. Both of the polarimetric decomposition features and the statistical characteristics are used to get the final segmentation result, which helps to increase the accuracy. Experimental results demonstrate the effectiveness of the proposed approach. Without any artificial supervision, the proposed approach can produce superior segmentation results than results obtained with random initialized variational approach and Wishart-H-a classification approach.
\end{abstract}

Keywords: PoISAR Image segmentation, Variational model, Primal-dual method, Polarimetric decomposition, Automatic initialization

\section{Introduction}

Segmentation is an important step for SAR image processing and interpretation. It can provide useful information for many SAR applications such as automatic target recognition, classification, crop monitoring and so on. SAR images are inherently corrupted by speckle noise, which degrades the quality of SAR data and makes the segmentation of SAR images difficult. Speckle filters, such as Lee filter and Frost filter, can be used to smooth the speckle noise before segmentation [1,2]. However, speckle reduction methods also cause information loss, which is undesired for further processing. Other methods based on wavelet transform and multi-scale analysis are also utilized for SAR image segmentation [3]. Nevertheless, there is no universal segmentation methods exist due to the variety and complexity of SAR images.

\footnotetext{
* Correspondence: zjcao@uestc.edu.cn

School of Electronic Engineering, University of Electronic Science and Technology of China, Chengdu, Sichuan 611731, People's Republic of China
}

The emergence of variational methods provides another sort of methods for SAR image segmentation. Using of variational models for image segmentation started from the application of Snakes model [4], and got a great advancement when Osher and Sethian put forward the level set method [5]. I. B. Ayed et al. [6] proposed a level set based SAR image segmentation method. The energy functional for SAR image segmentation is built based on the Gamma distribution, which can model the statistical property of SAR data. Then they extended their level set based SAR image segmentation approach to deal with PolSAR image segmentation [7]. PolSAR images are different from single-channel SAR images in the data storage form. The vector or matrix valued PolSAR data brings new issues such as data modeling and computational complexity. In [7], the segmentation of PolSAR images is tackled by taking advantage of the scaled Wishart distribution of coherency matrix and a multi-phase level set approach. Although 
this methods shows effectiveness for PolSAR image segmentation, there are still spaces to explore. Firstly, the level set method is known to be time-consuming, especially for multi-phase image segmentation. Furthermore, the signed-distance function based representation makes the energy functional to be non-convex with respect to the level set functions, which causes energy optimization issues such as the existence of local minimum. Last but not the least, artificial supervision is still a crucial factor for obtaining satisfactory segmentation results for previous variational PolSAR image segmentation approaches. The number of clusters is often pre-determined by subjective observation. Due to the existence of local minimum, a good initialization is also in need because different initializations may lead to different segmentation results.

In order to deal with the aforementioned problems, in this paper a variational model based PolSAR image segmentation approach is presented. First of all, based on the continuous Potts model, the energy functional for PolSAR images is established by taking advantage of the scaled Wishart distribution of the coherency matrix. In this model, clusters are represented by indicator functions. Compared to level set based region representation, using of cluster indicator functions is more direct and clear. It also makes it possible to relax the energy functional to be convex, which is a great advantage for optimizing consideration. The scaled Wishart distribution based energy is minimized with a duality based approach, which has shown its efficiency for optimizing continuous Potts model based energies [8]. This approach can obtain global optimal value for the Potts model, which is a desired property for the segmentation of PolSAR images with complex scene. Nevertheless, in the approach of [8], the feature model parameters for every cluster should be estimated before the energy minimization is performed. The number of clusters also needs to be determined. This difficulty is tackled by using a polarimetric decomposition based automatic initialization technique [9]. Cloude and Pottier [10] proposed an unsupervised classification method by using the $H-a$ decomposition. The $H-a$ plane which represents the scattering mechanisms is divided into eight zones, resulting in the partition of terrains with different physical scattering characteristics. This method is unsupervised but the result is rough due to the effect of speckle noise. The output of this rough classification could be used as initialization conditions for other more sophistic classification methods. However, the optimal number of clusters is not always equal to eight. So another step is added to estimate the appropriate number of clusters. The automatic initializing step brings two benefits. Firstly, the number of clusters and the initialization statement for the segmentation are both determined by PolSAR data itself. As a result, the proposed PolSAR image segmentation approach is fully unsupervised. A good initialization also increases the efficiency of the segmentation process. Secondly, the proposed approach combines the polarimetric decomposition based classification method and the statistical based variational segmentation method together. This means different features are integrated into the proposed approach to determine the final segmentation result, which helps to increase the segmentation accuracy.

The outline of this paper is as follows: In Section 2, the proposed variational segmentation approach is presented, including the scaled Wishart distribution based Potts model and the duality based optimizing method. The $H-a$ decomposition based automatic initialization method is introduced in Section 3. In Section 4, the experiments are carried out by using the real polarimetric SAR datas collected by AIRSAR and UAVSAR. The effectiveness of the proposed PolSAR segmentation approach is verified. Results of several other methods, including the Wishart-H-a classification method and traditional variational segmentation method with random/artificial initialization, are also provided and compared with the result of the proposed approach. The paper is concluded in Section 5.

\section{The proposed segmentation model}

In this section, the energy functional for PolSAR image segmentation and the energy minimization approach is introduced. The energy functional is based on the continuous Potts model, but makes use of the scaled Wishart distribution, which is suitable for modeling the statistical property of PolSAR data. The established energy is minimized with a duality based method.

\subsection{Scaled Wishart distribution and maximum likelihood classification of PoISAR data}

The data of fully polarimetric SAR is collected and stored in the scattering matrix $\mathbf{S}$. Each element $S_{p q}$ represents the complex scattering coefficient of transmitting polarization state $p$ and receiving polarization state $q$. By using the Pauli decomposition, the scattering matrix $\mathbf{S}$ can be transformed into a scattering vector $\mathbf{k}$. For the reciprocal backscattering case $S_{h v}=S_{v h}$ the scattering vector can be written as

$$
\mathbf{k}=\frac{1}{\sqrt{2}}\left[\begin{array}{lll}
S_{h h}+S_{v v}, & S_{h h}-S_{v v}, & 2 S_{h v}
\end{array}\right]^{T}
$$

After multi-look processing, the coherency matrix is given as

$$
\mathbf{T}=\frac{1}{l} \sum_{i=1}^{l} \mathbf{k}_{i} \mathbf{k}_{i}^{* T}
$$


where $l$ is the number of looks, " is the complex conjugate and $\mathbf{k}$ is the $i-t h$ sample scattering vector.

Assuming that the scattering vector has a Gaussian distribution, the coherency matrix could have a scaled Wishart distribution with $n$ degrees of freedom, i.e.

$$
p(\mathbf{T} ; \mathbf{C})=\frac{n^{q n}|\mathbf{T}|^{n-q} \exp \left(-\operatorname{tr}\left(n \mathbf{C}^{-\mathbf{1}} \mathbf{T}\right)\right)}{K(n, q)|\mathbf{C}|^{n}}
$$

where $K(n, q)=\pi^{(1 / 2) q(q-1)} \Gamma(n) \cdots \quad \Gamma(n-q+1)$, tr is the operator to compute the trace of a matrix, the polarimetric dimension $q=3$ for the reciprocal case, and $\mathbf{C}$ is the cluster center coherency matrix.

The PolSAR data can be classified according to the maximum-likelihood (ML) criterion. Define the distance between one PolSAR data sample $\mathbf{T}$ and a cluster center $\mathrm{C}_{i}$ as:

$$
d_{m}\left(\mathbf{T}, \mathbf{C}_{i}\right)=\operatorname{In}\left|\mathbf{C}_{i}\right|+\operatorname{tr}\left(\mathbf{C}_{i}^{-1} \mathbf{T}\right)
$$

where $\mathbf{C}_{i}=\sum_{k=1}^{N_{i}} \mathbf{T}_{k} / N_{i}$ is the mean coherency matrix of the $i$-th cluster, $m$ is the number of clusters. The pixel belongs to the $i-t h$ cluster if

$$
d_{m}\left(\mathbf{T}, \mathbf{C}_{i}\right)<d_{m}\left(\mathbf{T}, \mathbf{C}_{j}\right), j=1, \ldots, m, j \neq i
$$

then the images can be classified by using equation (5) if the cluster center is given. However, the pixel based ML classification is easy to be affected by speckle noise. Variational segmentation models add another regularization term to overcome the impact of speckle noise.

\subsection{Scaled Wishart distribution based Potts model}

One of the widely used variational models for image segmentation is the Potts model [11] which was proposed by Potts in 1952. It can partition the continuous domain into disjoint subdomains by minimizing [8]:

$$
\begin{array}{r}
\min _{\left\{\Omega_{i}\right\}_{i=1}^{m}} E=\sum_{i=1}^{m} \iint_{\Omega_{i}} f_{i}(x, y) d x d y+\lambda \sum_{i=1}^{m}\left|\partial \Omega_{i}\right| \\
\text { s.t. } \bigcup_{i=1}^{m} \Omega_{i}=\Omega, \Omega_{k} \cap \Omega_{l} \neq \varnothing, k \neq l
\end{array}
$$

where each $f_{i}(x, y)$ is defined on $\Omega$ and expresses the characteristic of the $i-t h$ subdomain, $\left|\partial \Omega_{i}\right|$ expresses the length of the boundaries of subdomains $\Omega_{i}$.

Let function $u_{i}(x, y) \in[0,1]$ denotes the indicator function of disjoint subdomains $\Omega_{i}, i=1 \ldots m$. The function $f_{i}(x, y)$ is defined as the distance between one PolSAR data sample $\mathbf{T}(x, y)$ and the mean coherency matrix of the $i$-th region $\mathbf{C}_{i}$ :

$$
f_{i}(x, y)=d\left(\mathbf{T}, \mathbf{C}_{i}\right)=\operatorname{In}\left|\mathbf{C}_{i}\right|+\operatorname{tr}\left(\mathbf{C}_{i}^{-1} \mathbf{T}\right)
$$

Then the scaled Wishart distribution based Potts energy functional can be written as follows:

$$
\min _{u \in \Delta_{+}} E(u)=\sum_{i=1}^{m} \iint_{\Omega} u_{i}(x, y) f_{i}(x, y) d x d y+\lambda \sum_{i=1}^{m} \iint_{\Omega}\left|\nabla u_{i}\right| d x d y
$$

where

$$
\Delta_{+}=\left\{u_{i}(x, y) \mid \sum_{i=1}^{m} u_{i}(x, y)=1 ; u_{i}(x, y) \geq 0, i=1 \ldots m\right\}
$$

\subsection{Energy minimization by using a dual method}

E. Bae et al. [8] proposed a dual method to minimize the Potts model. We adopt this method to minimize our scaled Wishart distribution based segmentation energy for the sake of computational efficiency.

The minimization of the total variation (TV) term in (8) can be converted to a max problem $[12,13]$ :

$$
\lambda \iint_{\Omega}|\nabla u| d x d y=\max _{p \in C_{\lambda}}-\iint_{\Omega} \nabla u \cdot p d x d y=\max _{p \in C_{\lambda}} \iint_{\Omega} u d i v(p) d x d y
$$

where $p$ is an auxiliary variable. The discrete divergence operator div $: Y \rightarrow X$ is defined by $\operatorname{div}=-\nabla^{*}\left(\nabla^{*}\right.$ is the adjoint operator of $\nabla)$. For every $p \in Y$ and $u \in X$ we have $\langle-\operatorname{divp}, u\rangle_{X}=\langle p, \nabla u\rangle_{Y}$. If $Y$ and $X$ be the same set, the second equal sign in formula (10) is established. In the rest of this paper, the variable $u$ is called primal variable and $p$ is called dual variables [8]. According to the max-min theorem in references [14] and the constraint of $u_{i}(x, y)$ in formula (9), equation (8) can be changed into the following format, i.e. the dual model:

$$
\begin{aligned}
\max _{p_{i} \in C_{\lambda}} E(p)= & \iint_{\Omega}\left\{\operatorname { m i n } \left(f_{1}(x, y)+\operatorname{div}\left(p_{1}\right), \ldots, f_{m}(x, y)\right.\right. \\
& \left.\left.+\operatorname{div}\left(p_{m}\right)\right)\right\} d x d y
\end{aligned}
$$

where $C_{\lambda}=\left\{p: \Omega \rightarrow R^{2} \mid\|p\|_{2} \leq \lambda, p_{m \mid \partial \Omega}=0\right\}$ and $C_{\lambda}$ is a convex set.

As the dual model (11) is non-smooth and nondifferentiable, the minimization is difficult. To deal with this problem, E. Bae et al. [8] proposed an smooth approximation model. This approximation is based on the observation that one could use a function $g(u)$ to approximate an asymptotic function $\mathrm{g}_{\infty}$ via $g_{\infty}=$ $\lim _{s \rightarrow 0}\left\{g_{s}(z):=s g\left(s^{-1} z\right)\right\}$. Therefore the dual model (11) can be approximated by: 


$$
\max _{p_{i} \in C_{\lambda}} E_{s>0}(p)=-s \iint_{\Omega}\left\{\log \sum_{i=1}^{m} \exp \left(\frac{-f_{i}-\operatorname{div}\left(p_{i}\right)}{s}\right)\right\} d x d y
$$

The smooth Potts model (12) can be efficiently solved by a projected gradient algorithm. The basic idea of the projected gradient algorithm is that maximum the dual model (12) with respect to the variables $p_{i}, i=1, \ldots, m$ can be decomposed into two steps. At the first step, the variables $p_{i}, i=1, \ldots, m$ are updated by using the gradient-descend algorithm. Then they are projected into the domain $C_{\lambda}$. This algorithm can be summarized as Algorithm 1 [8]:

\section{Algorithm 1:}

(1) Let arbitrary time step-size $\delta>0, s>0, k=0, p_{i}^{0}=0$, $i=1, \ldots, m$, and get $u_{i}^{0}, i=1, \ldots, m$ from the automatic initialization method;

(2) Compute $\mathbf{C}_{i}$ on the basis of $u_{i}^{k}, i=1, \ldots, m$,

(3) Compute $f_{i}=f_{i}(x, y)=d\left(\mathbf{T}, \mathbf{C}_{i}\right)=\operatorname{In}\left|\mathbf{C}_{i}\right|+\operatorname{tr}\left(\mathbf{C}_{i}^{-1} \mathbf{T}\right)$,

(4) Update $p_{i}^{k+1},=1, \ldots, m$ by

$$
p_{i}^{k+1}=\operatorname{Proj}_{C_{\lambda}}\left(p_{i}^{k}+\delta \nabla u_{i}^{k}\right)
$$

(5) Let $k=k+1$, compute

$$
u_{i}^{k}=\frac{e^{-f_{i}-d i v\left(p_{i}^{k}\right)}}{\sum_{j=1}^{m} e^{\frac{-f_{j}-d i v\left(p_{j}^{k}\right.}{s}}}, i=1, \ldots, m
$$

Where Proj $j_{\mathrm{c}}$ is the projection operator to the convex set $C$;

(6) Repeat step (2)-(5) until some convergence criterion is satisfied;

(7) Finally, $u_{i}, i=1, \ldots, m$ is recovered by

$$
u_{l}=\left\{\begin{array}{cc}
1 & \text { if } l=\arg \min _{i=1, \ldots, m}\left(f_{i}+\operatorname{div}\left(p_{i}\right)\right) . \\
0 & \text { otherwise }
\end{array}\right.
$$

Because the energy incorporates the definition $f_{i}=f_{i}(x$, $y)=d\left(\mathbf{T}, \mathbf{C}_{i}\right)$, this algorithm is suitable for PolSAR image segmentation. However, this algorithm requires the initial value of $u_{i}, i=1, \ldots, m$ as input. Thus the initialization is a key issue for the segmentation. In the next section, an automatic initialization approach is introduced for initializing the segmentation process.

\section{Automatic initialization of the segmentation}

The initialization plays a crucial role in the segmentation of PolSAR images. As the number of clusters is an important parameter in segmentation [9], most segmentation methods about fully polarimetric SAR data use a predefined number of clusters. However, to determine the number of clusters by subjective observation is not suitable for processing huge amount of PolSAR data. In practice, it is preferred to determine the number of clusters automatically based on the property of fully polarimetric SAR data. There are several methods for adaptive clustering, which can select the optimal number of clusters [15-17]. For PolSAR data, the $H-\alpha$ decomposition [10] based classification methods can partition the data into 8 clusters in an unsupervised manner. Although the classification result is rough due to the impact of speckle noise, it can still provide a good initialization of other sophistic classification methods. However, the number of clusters is fixed to 8 , which is not always the truth for PolSAR images with various scenes. In the work of Cao et al. [9], the authors stated a method to get the appropriate number of clusters and the corresponding classification map. They use the SPAN/H/a/A initialization method to define the value of the $M$. In this paper, we just use the $\mathrm{H} / a$ initialization method to determine the value of $M$ and adopt the similar method to define the number of the clusters. The experiment results demonstrate that this initialization method of $M=8$ can also get a good segmentation results. In the next parts, the $H-\alpha$ decomposition and automatic initialization method will be presented.

\subsection{Cloude-pottier decomposition}

The Cloude-Pottier decomposition is based on the eigenvalue analysis of the polarimetric coherence matrix $\mathbf{T}$ [10]. Due to the matrix $\mathbf{T}$ is a positive semi-definite Hermite matrix, $\mathbf{T}$ can be decomposed into the following format:

$$
\mathbf{T}=\mathbf{U}_{3}\left[\begin{array}{ccc}
\lambda_{1} & 0 & 0 \\
0 & \lambda_{2} & 0 \\
0 & 0 & \lambda_{3}
\end{array}\right] \mathbf{U}_{3}^{H}
$$

where $\lambda_{i}, i=1,2,3$ are the eigenvalues of matrix $\mathbf{T}$. The matrix $\mathbf{U}_{3}$ can be written as:

$$
\mathbf{U}_{3}=\left[\begin{array}{ccc}
\cos \left(\alpha_{1}\right) e^{i \phi_{1}} & \cos \left(\alpha_{2}\right) e^{i \phi_{2}} & \cos \left(\alpha_{3}\right) e^{i \phi_{3}} \\
\sin \left(\alpha_{1}\right) \cos \left(\beta_{1}\right) e^{i \delta_{1}} & \sin \left(\alpha_{2}\right) \cos \left(\beta_{2}\right) e^{i \delta_{2}} & \sin \left(\alpha_{3}\right) \cos \left(\beta_{3}\right) e^{i \delta_{3}} \\
\sin \left(\alpha_{1}\right) \sin \left(\beta_{1}\right) e^{i \gamma_{1}} & \sin \left(\alpha_{2}\right) \sin \left(\beta_{2}\right) e^{i \gamma_{2}} & \sin \left(\alpha_{3}\right) \sin \left(\beta_{3}\right) e^{i \gamma_{3}}
\end{array}\right]
$$

where $\alpha_{i}, i=1,2,3$ is the targets scattering type, $\beta_{i}, i=1$, 2,3 is the targets azimuth angle, $\phi_{i}, \delta_{i}, \gamma_{i}, i=1,2,3$ are the targets phase angles.

Equation (16) can be further decomposed into

$$
\mathbf{T}=\lambda_{1} \mathbf{e}_{1} \mathbf{e}_{1}^{* T}+\lambda_{2} \mathbf{e}_{2} \mathbf{e}_{2}^{* T}+\lambda_{3} \mathbf{e}_{3} \mathbf{e}_{3}^{* T}
$$

where $\mathrm{e}_{i}$ are eigenvectors of matrix $\mathbf{T}$.

The definition of the entropy $H$ and angle $a$ are given as follows: 


$$
\left\{\begin{aligned}
H & =-\sum_{i=1}^{3} p_{i} \log _{3} p_{i} \\
\alpha & =\sum_{i=1}^{3} p_{i} \alpha_{i} \\
p_{i} & =\lambda_{i} / \sum_{i=1}^{3} \lambda_{i}
\end{aligned}\right.
$$

$$
\begin{aligned}
\widetilde{\mathbf{C}}_{i} & =\frac{1}{N_{i}} \sum_{l=1}^{N_{i}} \mathbf{T}_{l}, \widetilde{\mathbf{C}}_{j}=\frac{1}{N_{j}} \sum_{l=1}^{N_{j}} \mathbf{T}_{l}, \widetilde{\mathbf{C}} \\
& =\frac{1}{N_{i}+N_{j}} \sum_{l=1}^{N_{i}+N_{j}} \mathbf{T}_{l}
\end{aligned}
$$

then the likelihood-ratio can be expressed as:

$$
\Lambda_{i j}=\frac{L\left(\mathbf{C} \mid \phi_{i} \phi_{j}\right)}{L\left(\mathbf{C} \mid \phi_{i}\right) L\left(\mathbf{C} \mid \phi_{j}\right)}
$$

The entropy $H$ characterizes the randomness of the target scattering. When $H$ is low, the medium can be considered as weakly depolarizing, and a dominant scattering mechanism exists, which corresponds to the largest eigenvalue. When $H$ is high, the situation will be opposite and full eigenvalue spectrum should be consider. Especially when $H=1$, the scattering degrades to random noise and no information can be acquired by polarimetric observation.

Angle $\alpha$ is a continuous parameter ranging from $0^{0}$ to $90^{\circ}$ which represents the average targets scattering mechanism. The definitions of $\alpha_{i}, i=1,2,3$ are as formula (17). Specifically, when $\alpha=0^{\circ}$, the scattering mechanism can be considered as isotropic scattering. As $\alpha$ increases, the scattering mechanism will move from dipole scattering $\left(\alpha=45^{\circ}\right)$ to double bounced scattering $\left(\alpha>45^{\circ}\right)$, and finally reach to the dihedral mechanism $\left(\alpha=90^{\circ}\right)$.

\subsection{Merge the similar clusters}

To estimate the appropriate number of clusters, the similar clusters should be merged to given the classification map with different number of clusters. To determine if two clusters should be merged, the following hypothesis is made:

$$
\left\{\begin{array}{l}
H_{0}: \mathbf{C}_{i}=\mathbf{C}_{j} \\
H_{1}: \mathbf{C}_{i} \neq \mathbf{C}_{j}
\end{array}\right.
$$

Under $H_{0}$ hypothesis, it can be assumed that $\mathbf{C}_{i}=\mathbf{C}_{j}=$ $\mathrm{C}$ On the basis of formula (3), the likelihood function is:

$$
L\left(\mathbf{C} \mid \phi_{i} \phi_{j}\right)=\prod_{k=1}^{N_{i}} p\left(\mathbf{T}_{k} ; \mathbf{C}\right) \prod_{l=1}^{N_{j}} p\left(\mathbf{T}_{l} ; \mathbf{C}\right)
$$

Under $H_{1}$ hypothesis, it is

$$
L\left(\mathbf{C} \mid \phi_{i}\right) L\left(\mathbf{C} \mid \phi_{j}\right)=\prod_{k=1}^{N_{i}} p\left(\mathbf{T}_{k} ; \mathbf{C}_{i}\right) \prod_{l=1}^{N_{j}} p\left(\mathbf{T}_{l} ; \mathbf{C}_{j}\right)
$$

Where $C_{\mathrm{i}}$ and $\mathbf{C}_{\mathrm{i}}$ are the $\mathrm{i}$-th andj-th cluster center coherency matrices respectively, $\mathrm{N}_{i}$ and $\mathrm{N}_{i}$ are the number of data within $i$-th and $j-$ th cluster respectively, and $i \neq j$. Let:
Taking the logarithm of formula (24), and ignoring the constant terms, the distance measure $D_{m}\left(\mathbf{C}_{i}, \mathbf{C}_{j}\right)$ between $\mathbf{C}_{\mathrm{i}}$ and $\mathbf{C}_{i}$ at the $m-t h$ iteration can be given by

$$
\begin{aligned}
D_{m}\left(\mathbf{C}_{i}, \mathbf{C}_{j}\right) & =-\frac{1}{n} \operatorname{In} \Lambda_{i j} \\
& =\left(N_{i}+N_{j}\right) \operatorname{In}|\widetilde{\mathbf{C}}|-N_{i} \operatorname{In}\left|\widetilde{\mathbf{C}}_{i}\right|-N_{j} \operatorname{In}\left|\widetilde{\mathbf{C}}_{j}\right|
\end{aligned}
$$

Then the two clusters with the minimum distance $D_{m}$ $\left(\mathbf{C}_{i}, \mathbf{C}_{j}\right)$ can be merged. Perform the cluster merging procedure recursively until the number of clusters is equal to the permitting minimal number of clusters.

\subsection{Estimating the number of clusters}

With the classification result of different number of clusters, the optimal number of clusters can be selected as following. Let $m$ be the current number of clusters. Giving a cluster $\phi_{i}$ and the corresponding cluster center $\mathbf{C}_{i}$, in accordance with formula (3), the probability density function of $\mathbf{T}$ can be written as

$$
p\left(\mathbf{T} ; \phi_{i}\right)=\frac{n^{q n}|\mathbf{T}|^{n-q} \exp \left(-\operatorname{tr}\left(n \mathbf{C}_{i}^{-1} \mathbf{T}\right)\right)}{K(n, q)\left|\mathbf{C}_{i}\right|^{n}}
$$

For $m$ clusters, the mixture probability density function is:

$$
p\left(\mathbf{T} ; \Phi_{m}\right)=\sum_{i=1}^{m} p(i) p\left(\mathbf{T} ; \phi_{i}\right)
$$

where $p(i)=N_{i} / N, N_{i}$ is the number of data within cluster $\phi_{i}, N$ is the number of the whole data.

Denote $X=\left\{\mathbf{T}_{1}, \ldots, \mathbf{T}_{N}\right\}$ the whole set of sample coherency matrices, the $m$-th data log-likelihood can be given by:

$$
L_{m}(X)=\operatorname{In}\left(\prod_{l=1}^{N} p\left(\mathbf{T}_{l} ; \Phi_{m}\right)\right)
$$

By using the equations (26), (27) and (28) and omit the constant terms, the log-likelihood becomes: 


$$
L_{m}(X)=\sum_{l=1}^{N} \operatorname{In}\left(\sum_{i=1}^{m} p(i) \exp \left(-n \cdot d\left(\mathbf{T}_{l}, \mathbf{C}_{i}\right)\right)\right)
$$

The value of $L_{m}(X)$ is depend on $m$ only, and $L_{m}(X)$ is a non-decreasing function about $m$. As a result the number of clusters cannot be estimated by $L_{m}(X)$ However, the change in the value of $L_{m}(X)$ provides useful information for determine the number of clusters. The change of the data log-likelihood can be measured by:

$$
D_{m}(X)=L_{m+1}(X)-L_{m}(X)
$$

Then, the optimum clusters can be decided by using the following expression:

$$
m_{\text {opt }}=\min \left\{m \mid D_{m}(X) \leq \varepsilon\right\}, m=1, \ldots, M-1
$$

Where $\varepsilon$ is a small threshold. In the work of Cao et al. [9], $\varepsilon$ is determined in an automatic way. Here, the value of $\varepsilon$ is set in an experiential way, but it can still lead to a reasonable result. Then the appropriate number of clusters is obtained with the minimum $D_{m}(X)$.

In this paper, the value of $M$ is 8 as the $H-a$ classification is used. The classification map corresponding to the optimal number of clusters can be also obtained. Then they are feed into the variational segmentation block, which is taken as the initializing condition for the iteration minimization process. The whole process of the proposed PolSAR segmentation process is demonstrated in Figure 1.

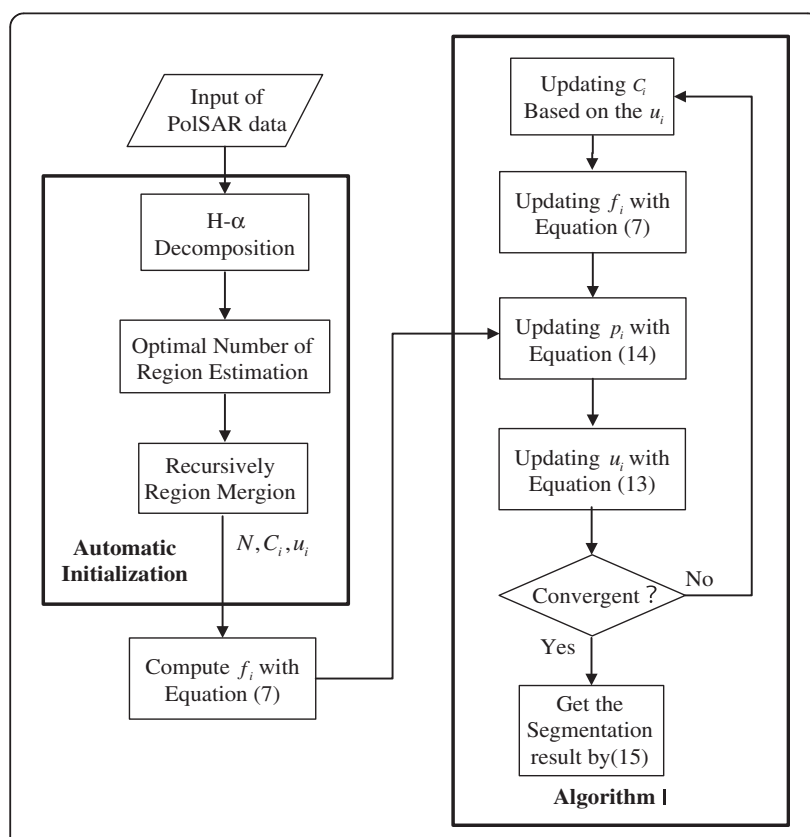

Figure 1 The flow chart of the algorithm.

\section{Experimental results}

Real PolSAR datas are taken to test the proposed approach. We take the AIRSAR data and UAVSAR data as examples to verify the effectiveness of our method. These data sets are part of real fully PolSAR data sets that collected by the AIRSAR and UAVSAR respectively. The types of both datas are multi-look, and the sizes are $400 \times 600$ for the two data sets. We don't use the speckle filter to preprocess them. For the two data sets, we set the threshold as $\varepsilon=1 e-10$. When the number has been determined, the automatic initialization can be obtained on the basis of the Section 3. Moreover, the results validate the effectiveness of the automatic initialization approach.

\subsection{Segmentation results of AIRSAR data}

For the AIRSAR data (Flevoland area), the $D_{m}(X)$ is drawn in Figure 2(a). The chart shows that the optimal number of clusters is seven. Figure 3 presents the segmentation results of Flevoland area. The power image and the Wishart-H- $a$ classification result are put in Figure 3(a) and in Figure 3(b) respectively, to make us compare conveniently. In Figure 3(c), one primal variable is set as the background, that is the black cluster. The other 6 primal variables are random circles with other six colors. Figure $3(\mathrm{~d})$ is the corresponding segmentation result, which is rough due to the unreasonable initialized cluster centers. In Figure 3(d), the orange cluster and green cluster occupy the most areas of the image, some other colors have embedded in the orange cluster and some areas have been mixed with several colors. Thus, the segmentation result in Figure 3(d) shows that this initializing method could not get a reasonable result. In Figure 3(e), 6 primal variables are initialized by manual quadrangles and the last one is initialized as the black background. The result is shown in Figure 3(f). In Figure 3(f), the white cluster has some pixels that belong to the light green cluster, and some clusters do not have smooth edges. Even though it is realizable to segment the PolSAR data into seven clusters in a general way, the manual quadrangles contain the artificial prior knowledge which is assumed by people. The automatic initialization based on the scaled Wishart distribution and $H-a$ decomposition is in Figure 3(g), and Figure 3(h) is the corresponding result. The proposed approach unites scattering characteristic with statistical characteristic, resulting in a better segmentation result when there is no a priori knowledge. Clusters are well separated in Figure 3(h) with little cluster mixing phenomenon. The edges are also smooth.

\subsection{Segmentation results of UAVSAR data}

For the UAVSAR data, from the colored span image that shown in Figure 4(a), it can be observe that this scene is 


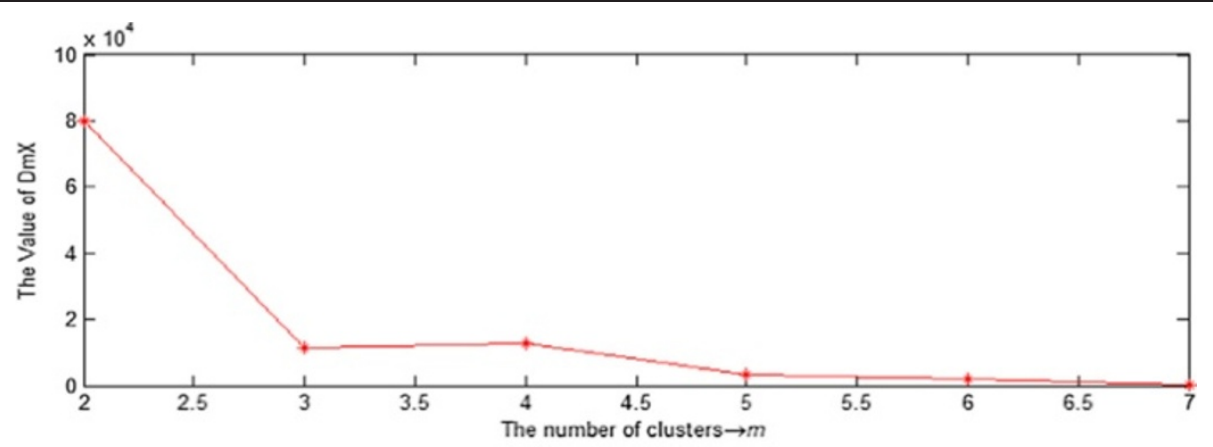

(a)

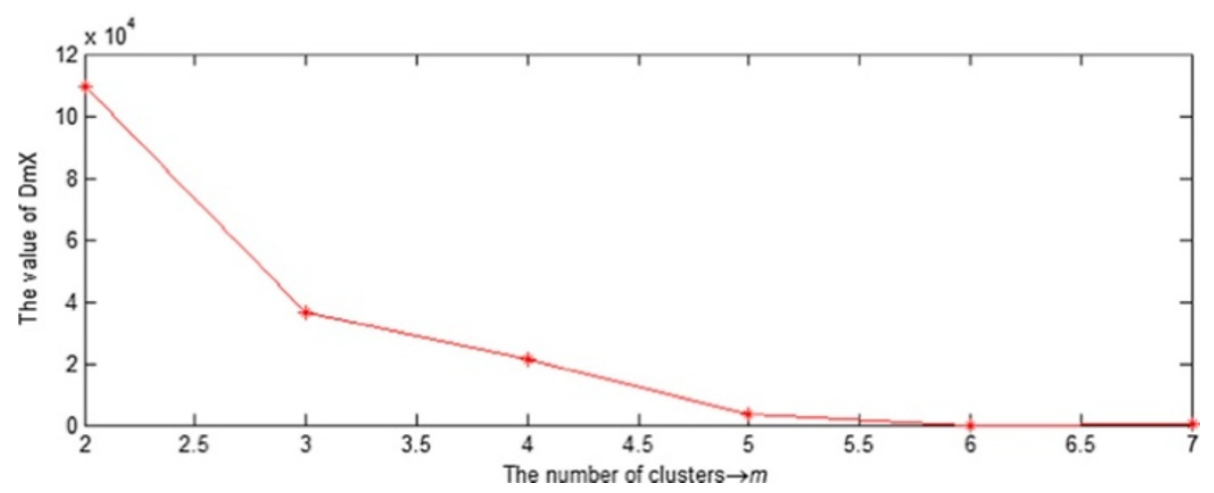

(b)

Figure $2 D_{m}(X)$ of PolSAR data (a) AIRSAR data, (b) the UAVSAR data.

covered by 5 or 6 terrain types. In Figure 2(b), the value of $D_{m}(X)$ with different number of clusters is shown. We can determine the optimal number of clusters is 6 on the basis of the automatic initialization procedure. It is expected to segment this data into 6 clusters, i.e. the white forest, green forest, dark green fields, light green fields, dark red-brown fields, and blue fields. The segmentation results of the proposed method and several other PolSAR image segmentation methods are given in Figure 4. In Figure 4(b), the segmentation result of the Wishart-H-a method is presented. The impact of speckle noise can be clearly observed. Many pixels in the cluster of green forest are misclassified. The segmentation result obtained by using the variational model with various initialization conditions are also given in Figure 4 (c)-(h). The initialization in Figure 4(c) of primal variables is set to be some circles, which leads that the cluster center coherency matrices are set unreasonable. It can be observed that the segmentation result is poor, as the Figure 4(d) explains. There are some clusters have been mixed, like the black cluster and white cluster. We can't distinguish the white forest from the green forest. In Figure 4(e), the initialization of primal variable is set artificially as some irregular polygons, the corresponding result is given in Figure 4(f). Compared to the result in
Figure 4(d), the segmentation is smoother because the cluster center coherency matrices are set artificially in a supervised way. All 6 clusters are well distinguished. But the segmentation result shows that some part of the dark green fields has been divided into the blue fields or the light green fields. The comparison of Figure 4(d) and Figure 4(f) clearly show the effect of the initial conditions on the final segmentation result. So a reasonable initialization is in need. The automatic initialization on the basis of $H-a$ decomposition is as Figure 4(g). The initial value of $M$ is 8 . According to the formula (31) and the estimated number of $m$, the minimum $D_{m}\left(\mathbf{C}_{i}\right.$, $\mathrm{C}_{j}$ ) should be merged until $\mathrm{m}=6$. The segmentation result is in Figure 4(h), which is close to the result in Figure 4(f). This demonstrated the effectiveness of the proposed automatic initialized variational segmentation approach. Moreover, in clusters which have complex scattering mechanism the power image, the segmentation result of the proposed approach can discriminative different scattering mechanisms better than the result in Figure 4(f).

These results show that the proposed approach is more convictive than the traditional variational segmentation and Wishart-H-a classification approach for segmentations of PolSAR images in a completely unsupervised way. 


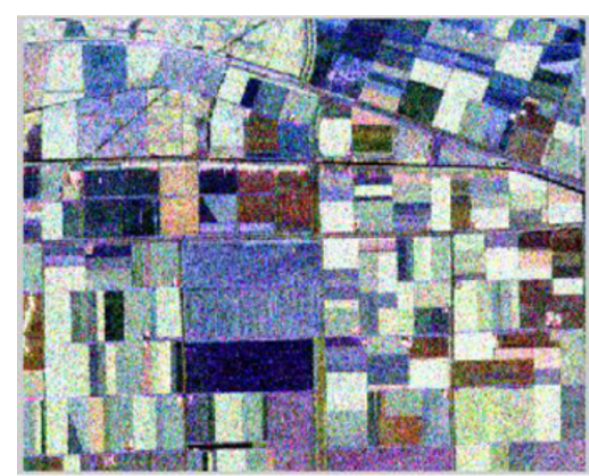

(a)

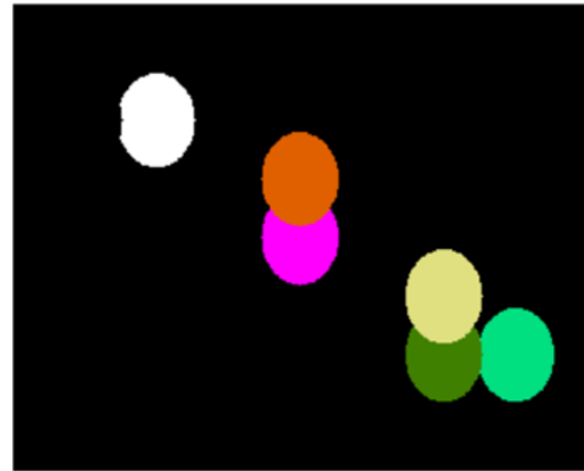

(c)

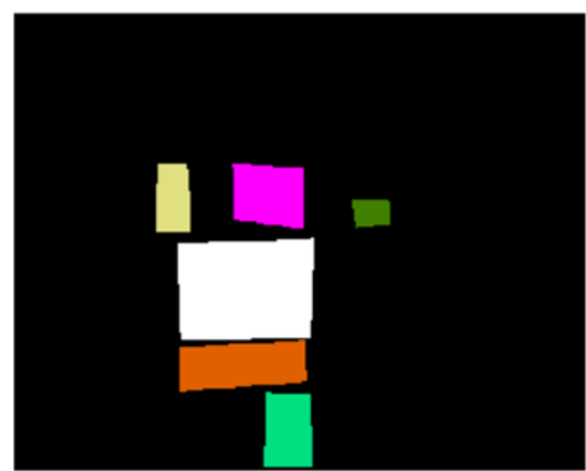

(e)

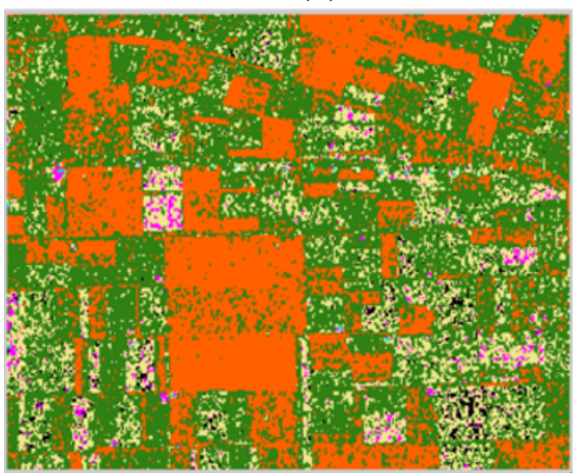

(g)

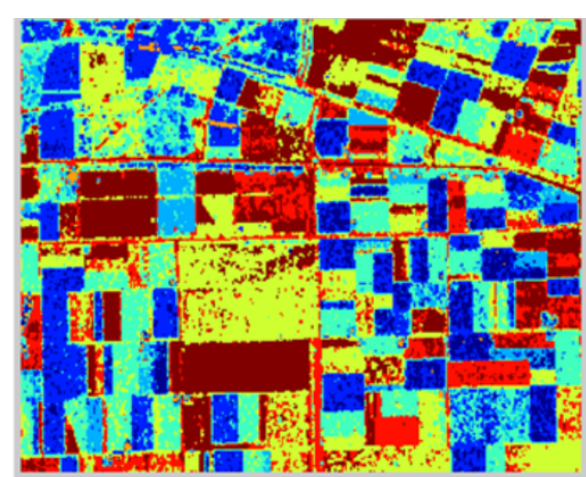

(b)

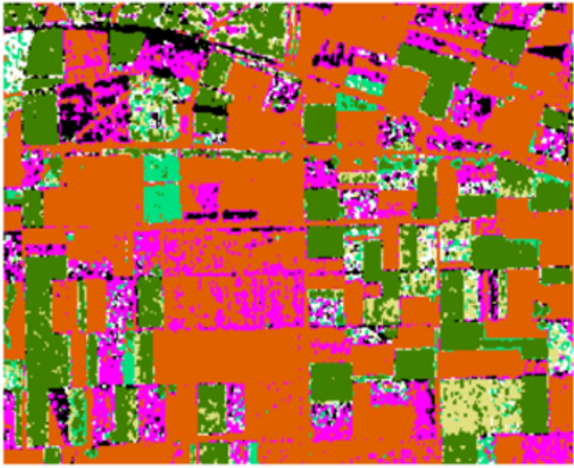

(d)

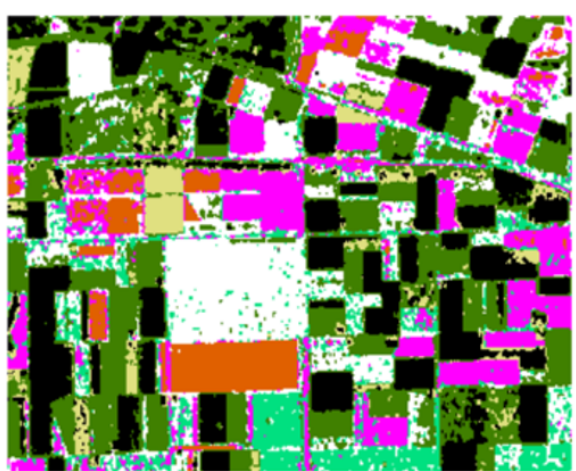

(f)

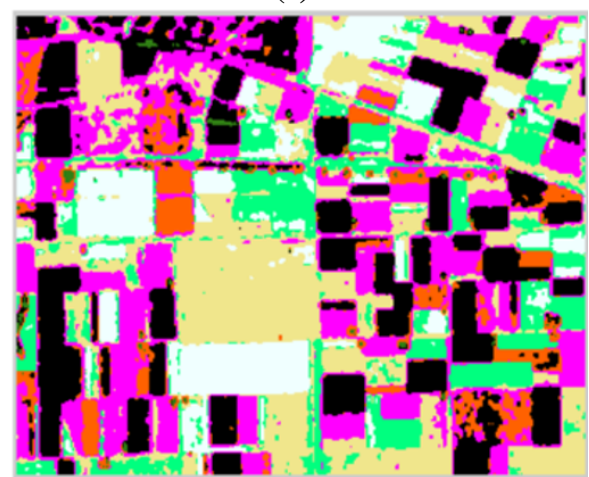

(h)

Figure 3 Segmentation result on an AIRSAR image (a) the power image, (b) the Wishart-H-a classification, (c) the random initialization, (d) segmentation result corresponding to (c), (e) initialization by manual quadrangles, (f) segmentation result corresponding to (e), (g) the automatic initialization, (h) segmentation result of our approach. 


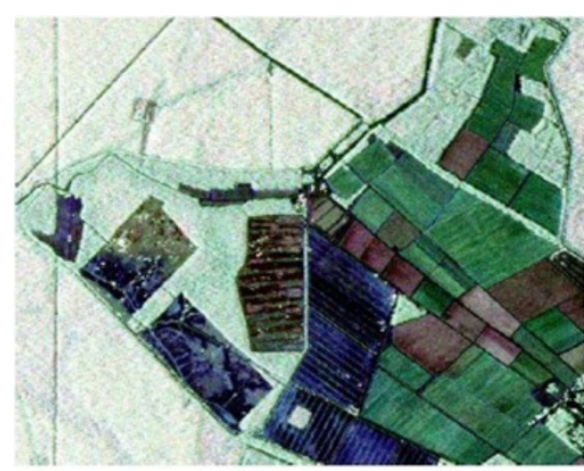

(a)

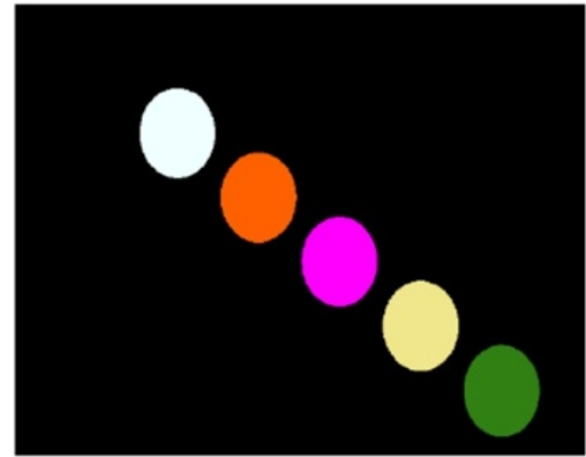

(c)

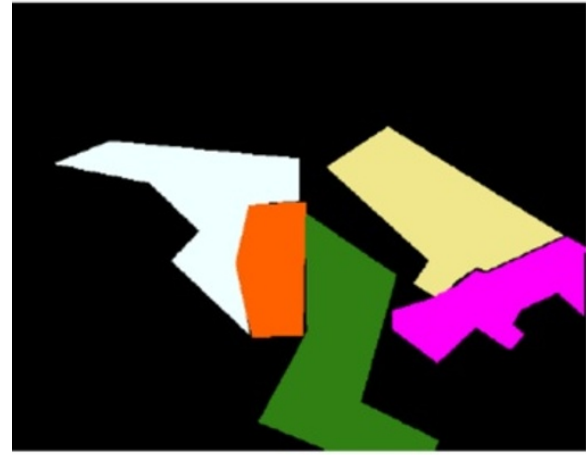

(e)

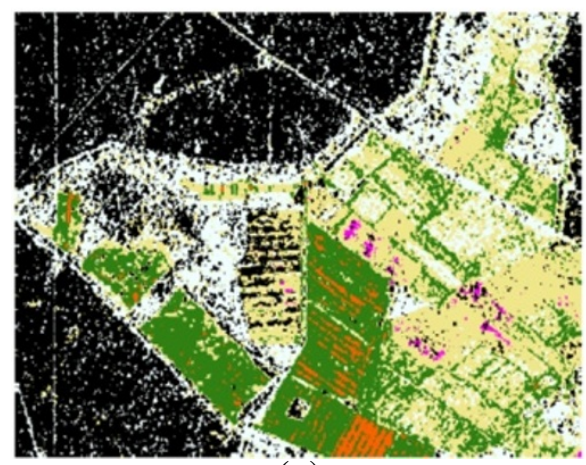

(g)

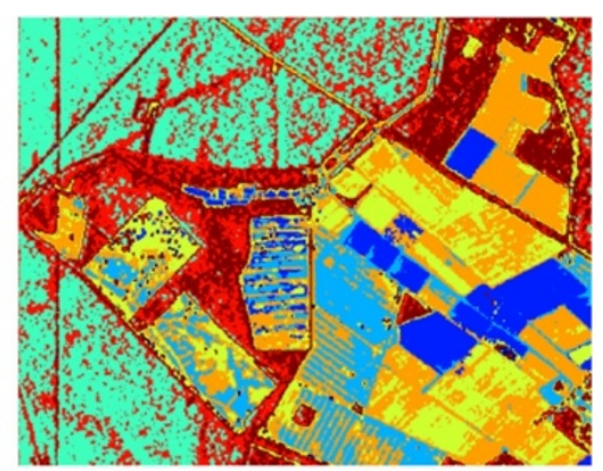

(b)

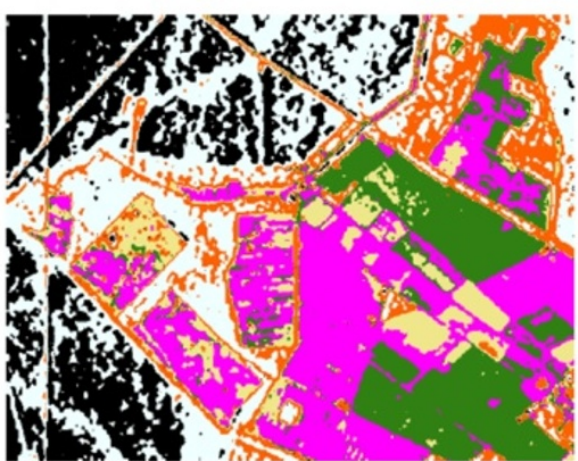

(d)

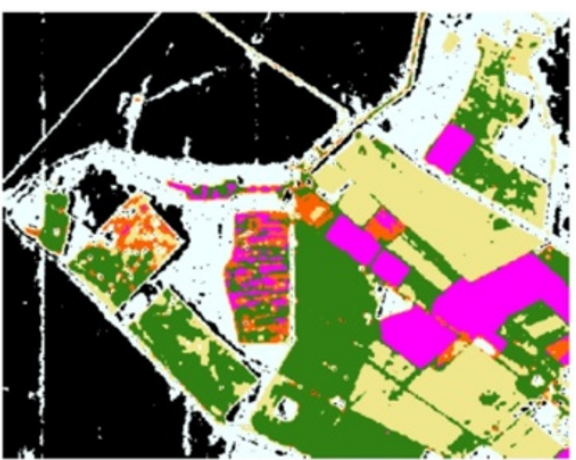

(f)

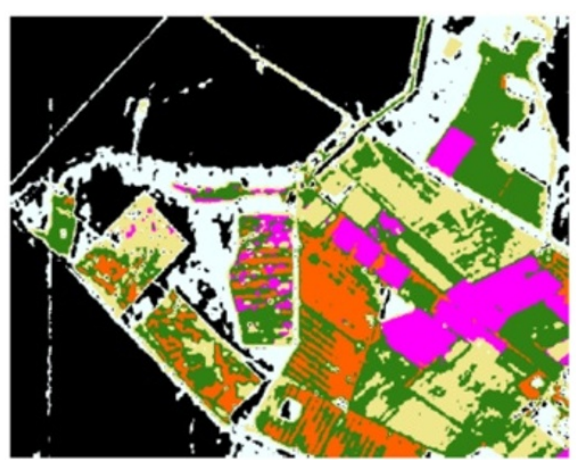

(h)

Figure 4 Segmentation result on a UAVSAR image (a) the power image, (b) the Wishart-H-a classification, (c) the random initialization, (d) segmentation result corresponding to (c), (e) initialization by manual quadrangles, (f) segmentation result corresponding to (e), (g) the automatic initialization, (h) segmentation result of our approach. 


\section{Conclusion}

This paper proposed a novel dual model and an automatic initialization approach for the PolSAR image segmentation problems. Since we employ the projected gradient algorithm to segment images, we could get the segmentation results of PolSAR images in a short time. Besides, the results of random initialization and manual quadrangles initialization declare that inappropriate initializations would generate unsatisfactory segmentation results. The automatic initialization includes scaled Wishart distribution and $H-a$ decomposition, which combine scattering characteristic with statistical characteristic, giving rise to a better segmentation result. The traditional method don't use the scattering characteristic, the results in Section 4 show that our automatic algorithm outperforms the general initialization under the condition of unsupervised.

\section{Competing interests}

The authors declare that they have no competing interests.

\section{Funding}

This work is supported by the Fundamental Research Funds for the Central Universities under Projects ZYGX2009Z005 and the National Natural Science Foundation of China under Projects 61271287.

Received: 24 December 2012 Accepted: 16 January 2013

Published: 19 February 2013

\section{References}

1. JS Lee, MR Grunes, SA Mango, Speckle reduction in multi-frequency and multipolrizationsarimagery. IEEE Trans. Geosci. Remote Sens. 29, 534-544 (1997)

2. J Xiao, J Li, A Moody, A detail-preserving and flexible adaptive filter for speckle suppression in SAR imagery. Int. J. Remote Sens. 24, 2451-2465 (2003)

3. E Mingolla, A neural network for enhancing boundaries and surface synthetic aperture radar images. Neural Networks 12(3), 499-511 (1999)

4. M Kass, A Witkin, D Terzopoulos, Snakes: Active contour models. Int. J. Comput. Vis. 4(1), 321-331 (1987)

5. S Osher, J Sethian, Fronts propagating with curvature dependent speed: algorithms based on the hamilton-jacbion formulation. Journal of Computational Physics 79(1), 12-49 (1988)

6. IB Ayed, A Mitiche, Z Belhadj, Multiregion level-set partitioning of synthetic aperture radar images. IEEE Trans. Pattern Analysis and Machine Intelligence 27, 793-800 (2005)

7. B Ayed, A Mitiche, Z Belhadj, Polarimetric image segmentation via maximum- likelihood approximationand efficient multiphase level-sets. IEEE Trans. Pattern Analysis and Machine Intelligence 28, 1493-1500 (2006)

8. E Bae, J Yuan, XC Tai, Global minimization for continuous multiphase partitioning problems usingdual approach. Int. J. Comput Vis. 92(1), 112-129 (2011)

9. F Cao, W Hong, Y Wu, E Pottier, An unsupervised segmentation with an adaptive number of clusters using the span/h/a/a space and the complex wishart clustering for fully polarimetric sar data analysis. IEEE Trans. Geosci. Remote Sens. 45, 3454-3467 (2007)

10. SR Cloude, E Pottier, An entropy based classication scheme for land applications of polarimetric SAR. IEEE Trans. Geosci. Remote Sens. 35(1), 68-78 (1997)

11. RB Potts, Some generalized order-disorder transformations. Proceedings of the Cambridge PhilosophicalSociety 48, 106-109 (1952)

12. A Chambolle, An algorithm for total variation minimization and applications. Journal of Mathematicallmaging and Vision 20(1-2), 89-97 (2004)

13. Y Meyer, Oscillating Patterns in Image Processing and Nonlinear Evolution Equations (The Fifteenth Dean Jacqueline B. Lewis Memorial Lectures, Boston, MA, USA: American Mathematical Society, 2001)
14. I Ekeland, R Teman, Convex Analysis and Variational Problems (PA. USA, Society for Industrial and Applied Mathematics Philadelphia, 1999)

15. M Lehsaini, H Guyennet, M Feham, An efficient cluster-based selforganisation algorithm for wireless sensor networks. International Journal of Sensor Networks 7(1-2), 85-94 (2010)

16. AS Raghuvanshi, S Tiwari, R Tripathi, N Kishor, Optimal number of clusters in wireless sensor networks: a FCM approach. International Journal of Sensor Networks 12(1), 16-24 (2012)

17. B Singh, DK Lobiyal, An energy-efficient adaptive clustering algorithm with load balancing for wireless sensor network. International Journal of Sensor Networks 12(1), 37-52 (2012)

doi:10.1186/1687-1499-2013-34

Cite this article as: Cao et al:: Segmentation of PoISAR image by using an automatic initialized variational model and a dual optimization approach. EURASIP Journal on Wireless Communications and Networking 2013 2013:34

\section{Submit your manuscript to a SpringerOpen ${ }^{\circ}$ journal and benefit from:}

- Convenient online submission

- Rigorous peer review

- Immediate publication on acceptance

- Open access: articles freely available online

- High visibility within the field

- Retaining the copyright to your article

Submit your next manuscript at $>$ springeropen.com 